\title{
Radiation-induced crosslinking: III. Effect on the crystalline and amorphous density fluctuations of polyethylene")
}

\author{
C. J. Chen and G. S. Y. Yeh \\ Departments of Chemical Engineering and Metallurgical Engineering and the Macromolecular Research Center, \\ University of Michigan Ann Arbor, Michigan, USA
}

\begin{abstract}
Small-angle x-ray scattering (SAXS) was used to determine density fluctuation in radiation-induced crosslinked polyethylene of varying degrees of crystallinity. Density fluctuation FL decreases with increasing crystallinity, while it increases linearly with increasing radiation dose or degree of crosslinking. By means of extrapolation, density fluctuations in the crystalline and the amorphous phases $F L_{c}$ and $F L_{a}$ were obtained. At a given dose, $F L_{a}$ is greater than $F L_{c}$. The increase in $F L_{a}$ with radiation is found to be much greater than that of $F L_{\mathcal{C}}$ compared with the initial values at $0 \mathrm{Mrad}$, $F L_{c}$ showing only a negligible increase even at $312 \mathrm{Mrad}$. The present findings suggest that crosslinks are not introduced within the crystalline phase; they take place primarily in the noncrystalline phase, in agreement with the conclusions reached previously on the basis of changes in crystalline and amorphous densities in irradiated polyethylene.
\end{abstract}

Key words: Crosslinking; radiation; polyethylene; density; crystallinity; phase densities; density fluctuation; phase density fluctuations; $\bar{C}$ rosslink locations and mechanisms; defects; lateral grain boundaries.

\section{Introduction}

In previous papers $[1,2]$ the changes in densities of the crystalline and noncrystalline amorphous regions or phases of polyethylene (PE), with radiation were determined by means of SAXS. The results suggested that crosslinks take place primarily in the noncrystalline phase, in particular, at the defective regions, e.g., the lateral grain boundaries. In this paper we report changes in another structural parameter, density fluctuation of PE due to radiation, based on SAXS studies.

The SAXS intensity extrapolated to zero scattering angle has been related to the density fluctuation in a sample [3]. With this method the density fluctuation has been determined for several amorphous polymers $[4,5]$ and semicrystalline PEs [5] over a range of temperature. For PE the density fluctuation at room temperature was found to increase linearly with decreasing crystallinity, from 0.26 for a crystallinity of $85 \%$, to 0.51 for $43 \%$ crystallinity.

Density fluctuation within the phases of a sample causes an additional intensity component, producing deviations from Porod's law [6] in the high-angle region of SAXS. Based on this, Ruland [7] has shown that density fluctuation can be determined from slopes of suitable intensity plots. This was demonstrated by Perret and Ruland [8] for PE crystallized from both solution and melt. They showed that a three-dimensionally homogeneous fluctuation exists for PE, based on the linear relation of an $S^{3} I$ (s) vs $S^{3}$ plot, which was found to also hold for our samples. Therefore, this method is used in this study.

It has been shown in the case of natural rubber [5] that crosslinking by dicumyl peroxide resulted in an increased density fluctuation. Since radiation can introduce crosslinks in PE, radiation is also expected to cause increased density fluctuations. It is the pur-

*) Dedicated to Prof. Dr. W. Pechhold on the occasion of his 60 th birthday 
pose of this study to examine the changes in density fluctuation in irradiated PE and to determine the individual components of this fluctuation in the crystalline and amorphous phases in order to clarify further the location of crosslinks in PE suggested in the previous papers $[1,2]$.

\section{Theoretical background}

One of the basic assumptions in the theoretical treatment of the two-phase system is to consider the electron density within each phase as constant. This assumption holds for phases with a high degree of order and for voids. However, in actual cases the phases can be noncrystalline and/or crystalline having paracrystalline distortions, point defects, and dislocation lines, thereby invalidating the assumption. This density fluctuation within the phases leads to an additional component in the scattering intensity causing systematic deviations from Porod's law [6] at higher angles.

Assuming there is no correlation between the density fluctuation of one phase and that of the other, the corresponding intensity components are simply additive to the small-angle scattering of the ideal system. According to Ruland et al. $[7,8]$ the corrected scattered intensity is expressed as

$$
I(s)=\frac{V<\eta^{2}>}{2 \pi^{3} l_{c} s^{4}}+v_{1} \rho_{1} F L_{1}+v_{2} \rho_{2} F L_{2}
$$

or, for slit collimation:

$$
\tilde{I}(s)=\frac{V<\eta^{2}>}{4 \pi^{2} l_{c} s^{3}}+v_{1} \rho_{1} F L_{1}+v_{2} \rho_{2} F L_{2}
$$

where $s=2 \sin \Theta / \lambda, V$ is the irradiated volume, $\left\langle\eta^{2}\right\rangle$ is the mean-square density fluctuation $\left(=v_{1} v_{2}\left(\rho^{1}-\right.\right.$ $\left.\left.\rho_{2}\right)^{2}\right)$ and $l_{c}$ is the correlation length. $v_{1}, v_{2} \rho_{1}$ and $\rho_{2}$ are the volumes and densities of phase 1 and phase 2, and $F L_{1}$ and $F L_{2}$ are the intensity components of denstiy fluctuations within phase 1 and 2 , respectively.

For three-dimensional homogeneous fluctuation, $F L$ is given by $[3,7]$

$$
[F L]=F L_{3-\text { dimensional }}=\frac{\left\langle N^{2}\right\rangle_{v}-\langle N\rangle_{v}^{2}}{\langle N\rangle_{v}}
$$

where $N$ is the number of electrons and the average is taken over a volume which is large compared with the interaction between the atoms and molecules within a given phase. Density fluctuations of this type usually exist in liquids and gases. However, amorphous substances with "frozen-in" liquid structures [9] as well as crystalline substances with a statistical distribution of foreign atoms or vacancies will also show this type of density fluctuation.

In the case of strongly anisotropic disorder, however, the density fluctuations become inhomogeneous and $F L$ is no longer a constant. For example, if the disorder is one- or two-dimensional, fluctuations are given by [7]

$$
[F L]=\tau F L_{1-\operatorname{dim}} / 2 \pi S^{2}
$$

or

$$
[F L]=\xi F L_{2-\mathrm{din}} / 2 S,
$$

where $\tau$ is the surface density of electrons and $\xi$ is the linear density of electrons.

The separation of the scattering intensity due to density fluctuation from the scattering of an ideal two-phase system can be achieved by making a $S^{4} I$ $(s)$ vs $u$ plot in the case of a pinhole system, or an $S^{3}$ $\tilde{I}(s)$ vs $u^{\prime}$ plot in the case of a slit system of finite height [7]. The parameters $u$ and $u^{\prime}$ for the different types of fluctuations are given in Table 1. Having. found a linear relationship in one of these plots, the type of fluctuation is defined and the magnitude of the fluctuation is obtained from the slope. A linear relation in $S^{3} \tilde{I}$ vs $S^{3}$ was found to hold for our examples, indicating that the fluctuation in crosslinked $\mathrm{PE}$ to be three-dimensional in origin.

Table 1.

\begin{tabular}{lll}
{$[F L]$} & $u$ & $u^{\prime}$ \\
\hline One-dimensional & $s^{2}$ & $s^{2}$ \\
Two-dimensional & $s^{3}$ & $s^{3} \int_{0}^{\infty} W(z) / \sqrt{s^{2}+z^{2}} d z$ \\
Three-dimensional & $s^{4}$ & $s^{3}$ \\
\hline
\end{tabular}

\section{Experimental}

Four types of polyethylene (PE) supplied by DuPont Company, were studied: three linear (LPE), Alathon 7040, 7140, and 7440, and one branched (BPE), Alathon 1645. Pertinent information ( $\rho$, melt index, $M_{v}$ ) on these samples is summarized in Table 2. 
Table 2 .

\begin{tabular}{llll}
\hline Alathon & Density $\left(\mathrm{g} / \mathrm{cm}^{3}\right)$ & Melt Index & $\bar{M}_{v}$ \\
\cline { 2 - 4 } 7040 & 0.9508 & 6.0 & 41500 \\
7140 & 0.9439 & 4.0 & 45000 \\
7440 & 0.9360 & 4.0 & 48800 \\
1645 & 0.9220 & 4.5 & 34300 \\
\hline
\end{tabular}

Sample preparations and irradiation procedures were the same as those described in a previous paper [1]. Samples were subjected to radiation doses ranging from 10 to 312 Mrads. Procedures for gelation, bulk sample density and WAXS measurements were also given in the paper. As before [2], SAXS was carried out on a Rikagu-Denki camera with slit collimation.

\section{Results}

Changes in gel content as a function of radiation dose for each of the samples are shown in Fig. 1. Gel content increases with increasing dose for all the samples, resulting from the formation of a network structure during irradiation.

Changes in sample density (determined by density gradient column) as a function of radiation dose are

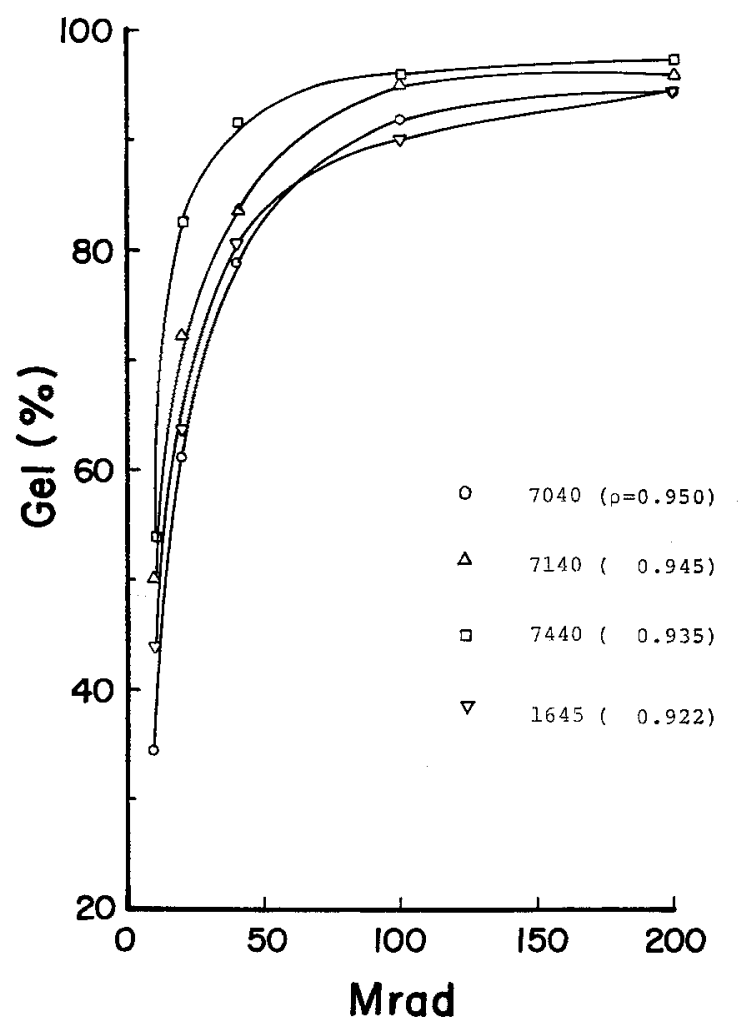

Fig. 1. Changes in gel content as a function of radiation dose

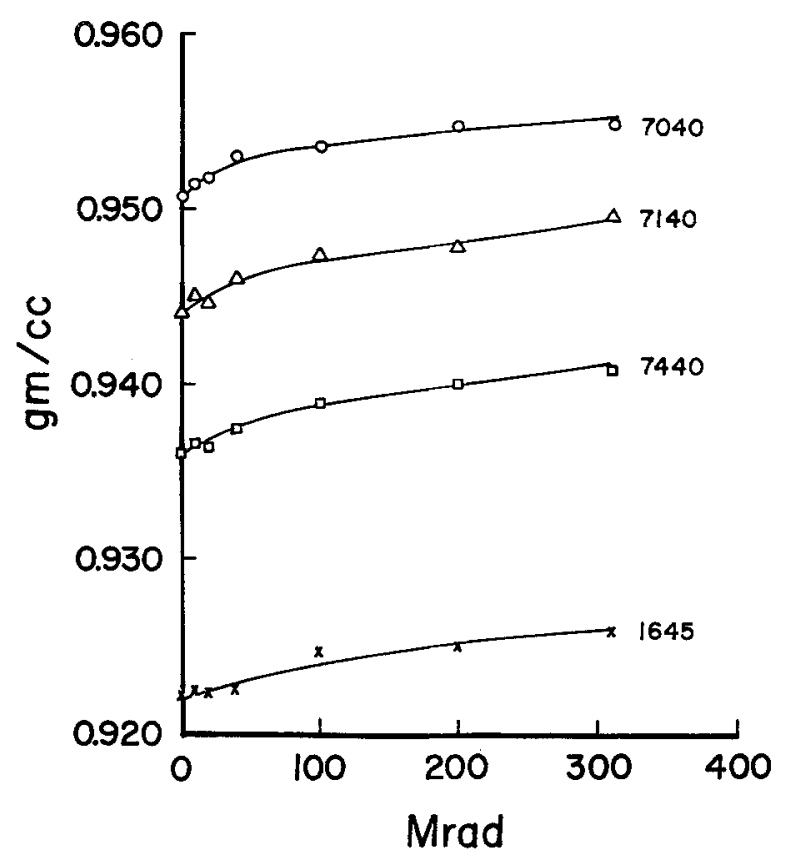

Fig. 2. Changes in density as a function of radiation dose

shown in Fig. 2. The density is seen to increase slightly with increasing dose for all the samples. Therefore, volume crystallinity $v_{c}$ calculated from the density data using constant $\rho_{c}$ and $\rho_{a}$ values increases with dose. However, crystallinity $w_{c}$ determined from WAXS, listed in Table 3, shows a decrease with increasing dose. The decrease is very gradual at small doses (up to $100 \mathrm{Mrad}$ ), after which the decrease becomes noticeably greater. For a discussion concerning the origin of the discrepancy between $v_{\mathcal{c}}$ and $w_{c}$ see $[1,2]$.

Table 3. Crystallinity

\begin{tabular}{|c|c|c|c|c|c|c|c|}
\hline Alathon $_{\text {Mrad }}$ & 0 & 10 & 20 & 40 & 100 & 200 & 312 \\
\hline 7040 & 75.9 & 75.7 & 75.4 & 75.7 & 74.9 & 74.6 & 73.5 \\
\hline 7140 & 72.4 & 72.0 & 70.4 & 71.6 & 72.8 & 71.0 & 70.0 \\
\hline 7440 & 63.0 & 63.0 & 62.7 & 62.9 & 63.0 & 62.5 & 61.1 \\
\hline 1645 & 51.8 & 51.9 & 51.0 & 51.5 & 51.9 & 51.1 & 47.1 \\
\hline
\end{tabular}

SAXS curves obtained from slit-collimation for each of the original unirrradiated samples are shown in Fig. 3. Peak intensity decreases greatly with decreasing sample density, while peak position tends 


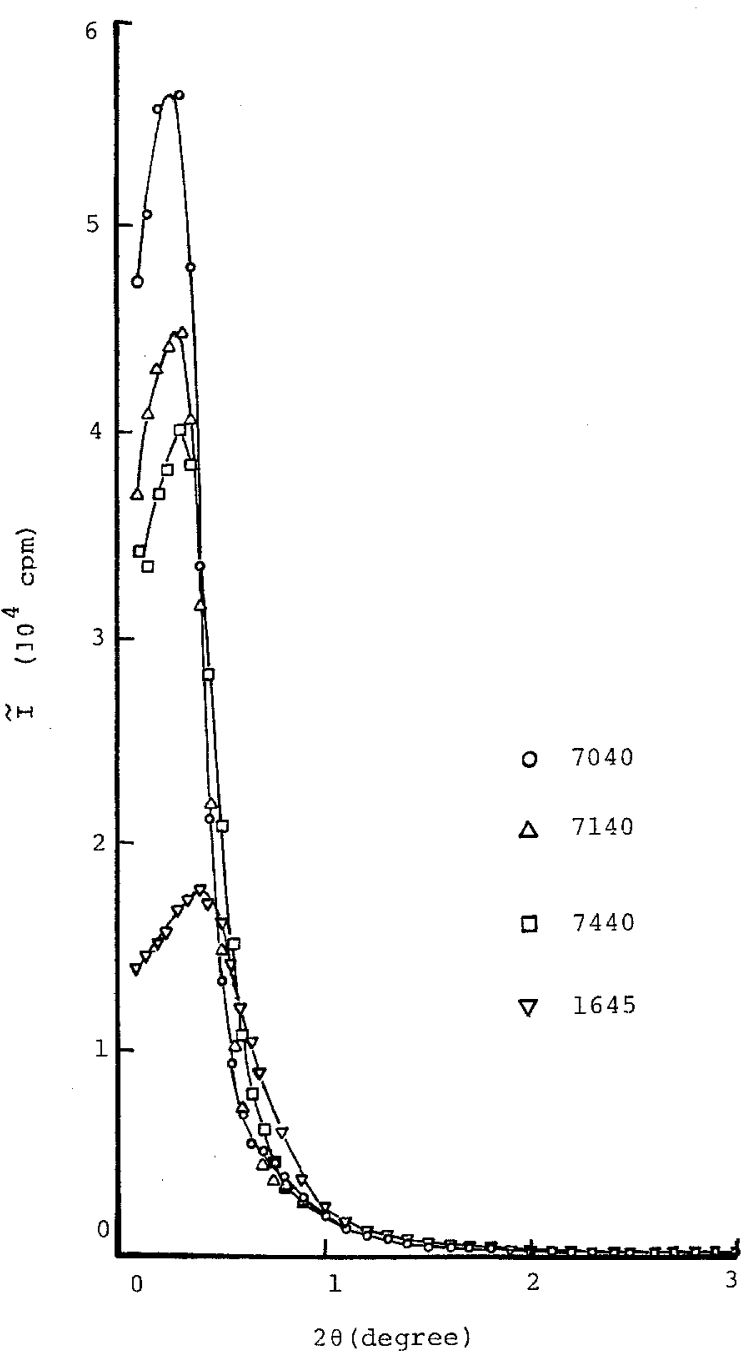

Fig. 3. SAXS curves of unirradiated PEs

to shift slightly to a higher angle. The long periods corresponding to peak positions are 300-350 $\AA$ for LPE and $220 \AA$ for BPE. Similar changes in peak intensity and long period are observed for all the irradiated samples. They are shown as examples in Figs. 4 and 5 at 100 and 312 Mrad, respectively.

The changes in SAXS curves with radiation are similar for all PE samples. The SAXS intensity decreases with increasing dose, while the peak position remains unchanged. Such curves are shown in Fig. 6 for a linear PE (Alathon 7040) and in Fig. 7 for a branched PE (Alathon 1645).

Figure 8 shows a $s^{3} \widetilde{I}(s)$ vs $s^{3}$ plot for the unir-

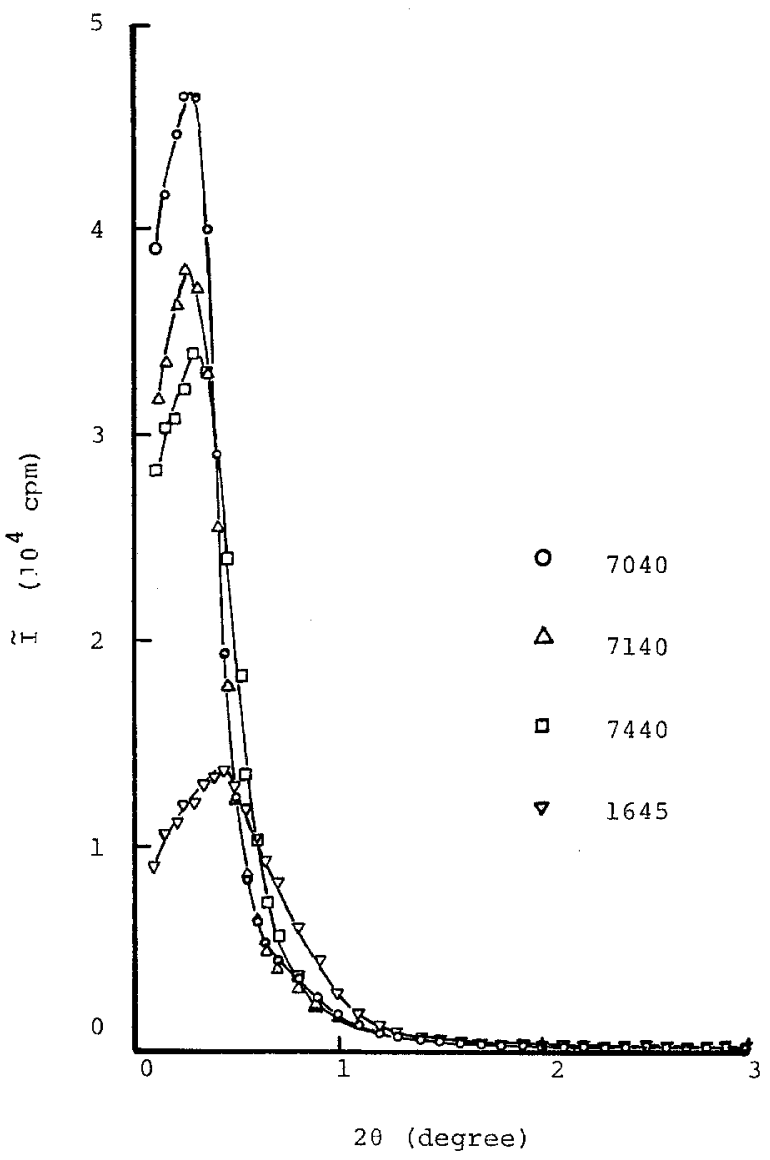

Fig. 4. SAXS curves of irradiated PEs (100 Mrad)

radiated samples. All the samples show deviations from Porod's law at large $s$ regions. The linear relation at large $s$ was obtained from the least-squares method. The straight lines fit the experimental data very well, as indicated by the high values of correlation coefficient between 0.98 and 0.99 . The results indicate that the slope increases with decreasing sample density, indicating an increase in density fluctuation with decreasing crystallinity. This is in agreement with the findings of Ratbje and Ruland [5]. Straight lines were also obtained for all the irradiated samples from the same plot. Plots for samples of 100 and 312 Mrad are shown in Figs. 9 and 10, respectively, where those points at lower $s$ regions are omitted for clarity. The observed changes in slope with sample density are similar to those of the unirradiated samples. 


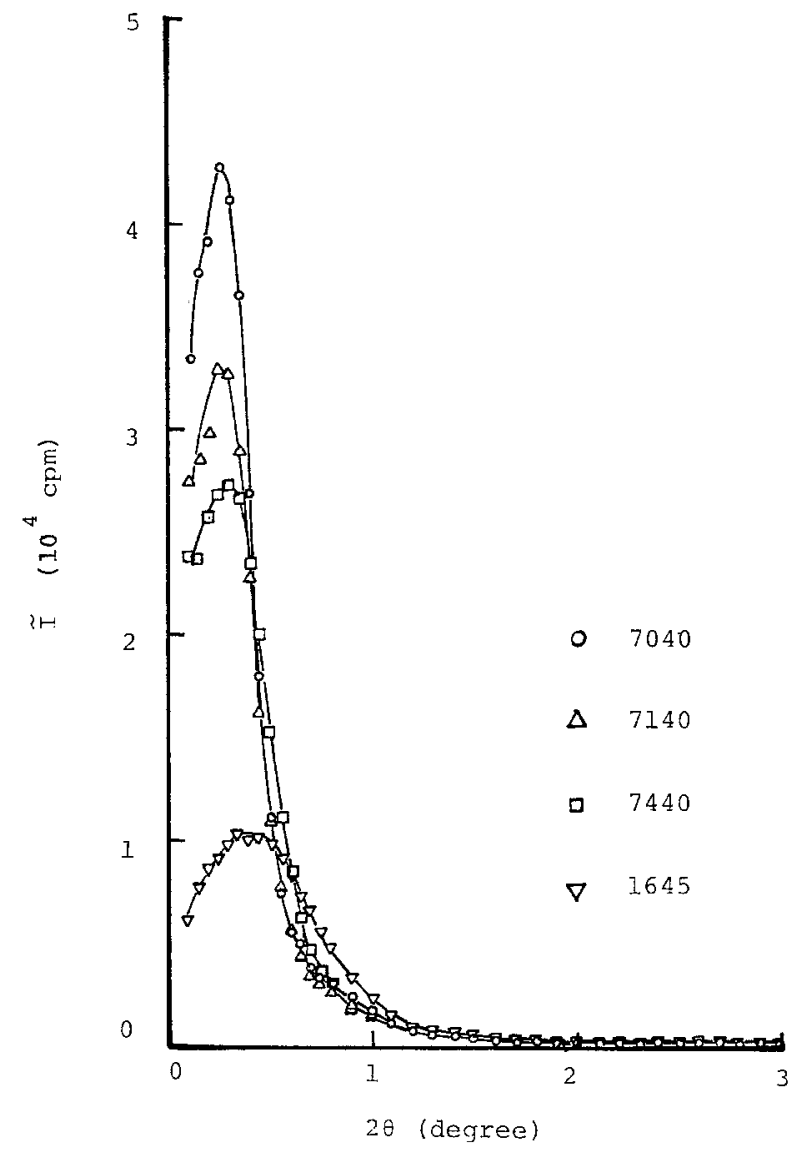

Fig. 5. SAXS curves of irradiated PEs (312 Mrad)

In order to show the changes in slope with radiation dose, the same $s^{3} \tilde{I}(s)$ vs $s^{3}$ plots are made for each type of sample. Examples are shown in Figs. 11 and 12 for Alathon 7040 and 1645, respectively. For each $P E$ the slope increases with increasing radiation dose, suggesting that density fluctuation increases with increasing dose or degree of crosslinking. The results also show that the intercept decreases with increasing dose, which indicates that $\left\langle\eta^{2}\right\rangle$ decreases with increasing degree of crosslinking, confirming the results obtained from the "invariant" in the previous paper [2]; this will be discussed later.

Density fluctuation $F L$ calculated from the slope was corrected for slit-length using Hendrick and Schmidt's program [10]. For unirradiated samples the $F L$ values are between 0.43 and 0.62 , in good agreement with the reported values of 0.26 and 0.51 $[5,8]$. Values of density fluctuation for all PEs of different doses are listed in Table 4. Figure 13 shows

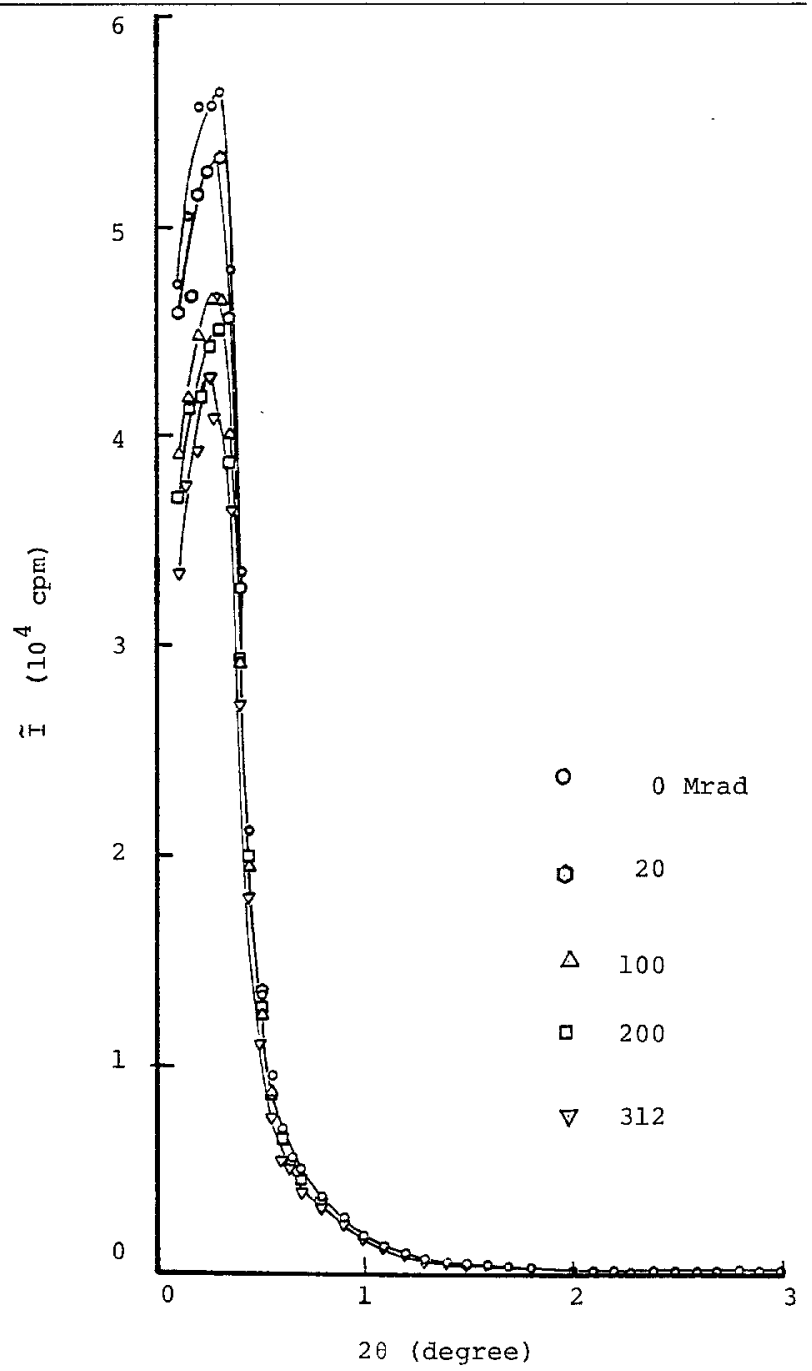

Fig. 6. SAXS curves of Alathon 7040 with various radiation doses

the change in density fluctuation as a function of radiation dose for all the samples. It can be seen that density fluctuation increases, not only with decreasing sample density, but also increases linearly with radiation dose.

Figure 14 shows a plot of density fluctuation $F L$ vs crystallinity $w_{c}$ (determined from WAXS). Linear relations were obtained for all samples, including one for the unirradiated samples, in agreement with the results of Ratbje and Ruland [5]. These results indicate that the density fluctuation in PE is composed of additive contributions from both the crystalline and amorphous phases. Therefore, the density fluctuation in the crystalline and the amorphous phases, $F L_{c}$ and $F L_{a}$, can be obtained by extrapolating $F L$ values to $w_{c}=1$ and $w_{c}=0$. For the unirradiated 


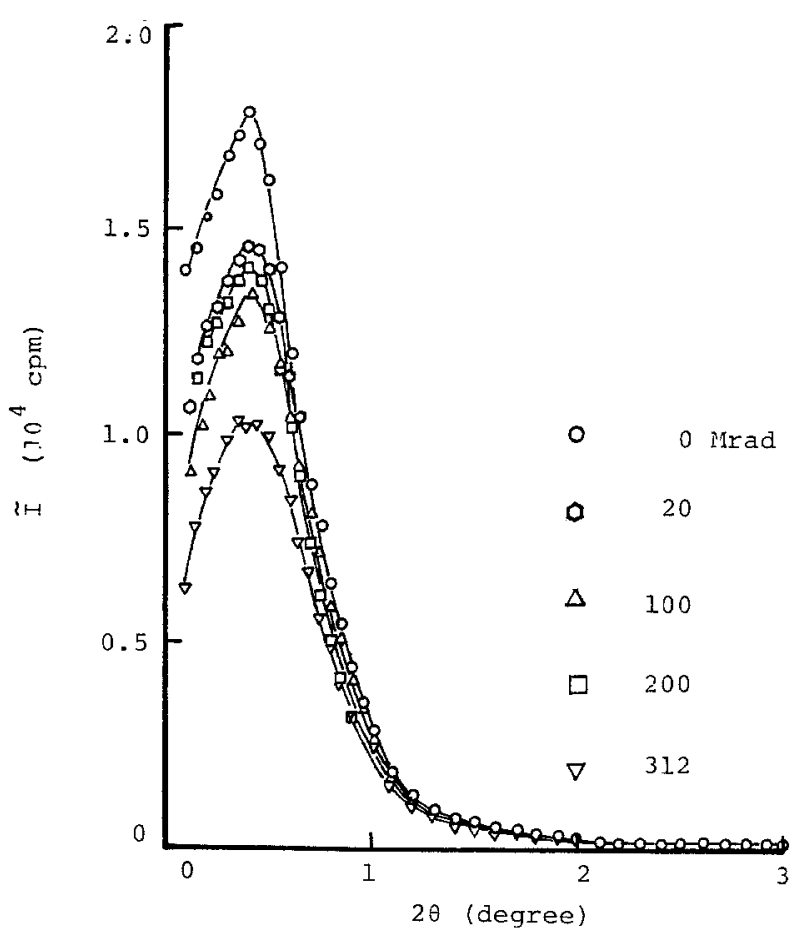

samples $F L_{c}$ and $F L_{a}$ are 0.23 and 1.04 , respectively. These values compare reasonably well with the values of 0.16 for $F L_{c}$ and 0.75 for $F L_{a}$ reported for PE [5]. Similarly, value of $F L_{c}$ and $F L_{a}$ were determined for each dose. The results indicate that the change in $F L_{a}$ is much greater than that in $F L_{c}$ when compared with the corresponding initial values at 0 Mrad. An increase of 0.47 (from 1.04 to 1.51) was obtained for $F L_{a}$ ad $312 \mathrm{Mrad}$, while only a negligible increase of 0.01 (from 0.23 to 0.24 ) was found for $F L_{c \text { r }}$ even at such a high dose.

Table 4. Density fluctuation

\begin{tabular}{|c|c|c|c|c|c|c|c|}
\hline Alathon & 0 & 10 & 20 & 40 & 100 & 200 & 312 \\
\hline 40 & 0.42 & 0.450 & 0.451 & 0.451 & 0.484 & 0.517 & 0.593 \\
\hline 7140 & 0.468 & 0.454 & 0.473 & 0.480 & 0.520 & 0.583 & 0.631 \\
\hline 7440 & 0.510 & 0.530 & 0.528 & 0.529 & 0.598 & 0.672 & 0.750 \\
\hline 1645 & 0.618 & 0.646 & 0.633 & 0.658 & 0.718 & 0.825 & 0.874 \\
\hline
\end{tabular}

Fig. 7. SAXS curves of Alathon 1645 with various radiation doses

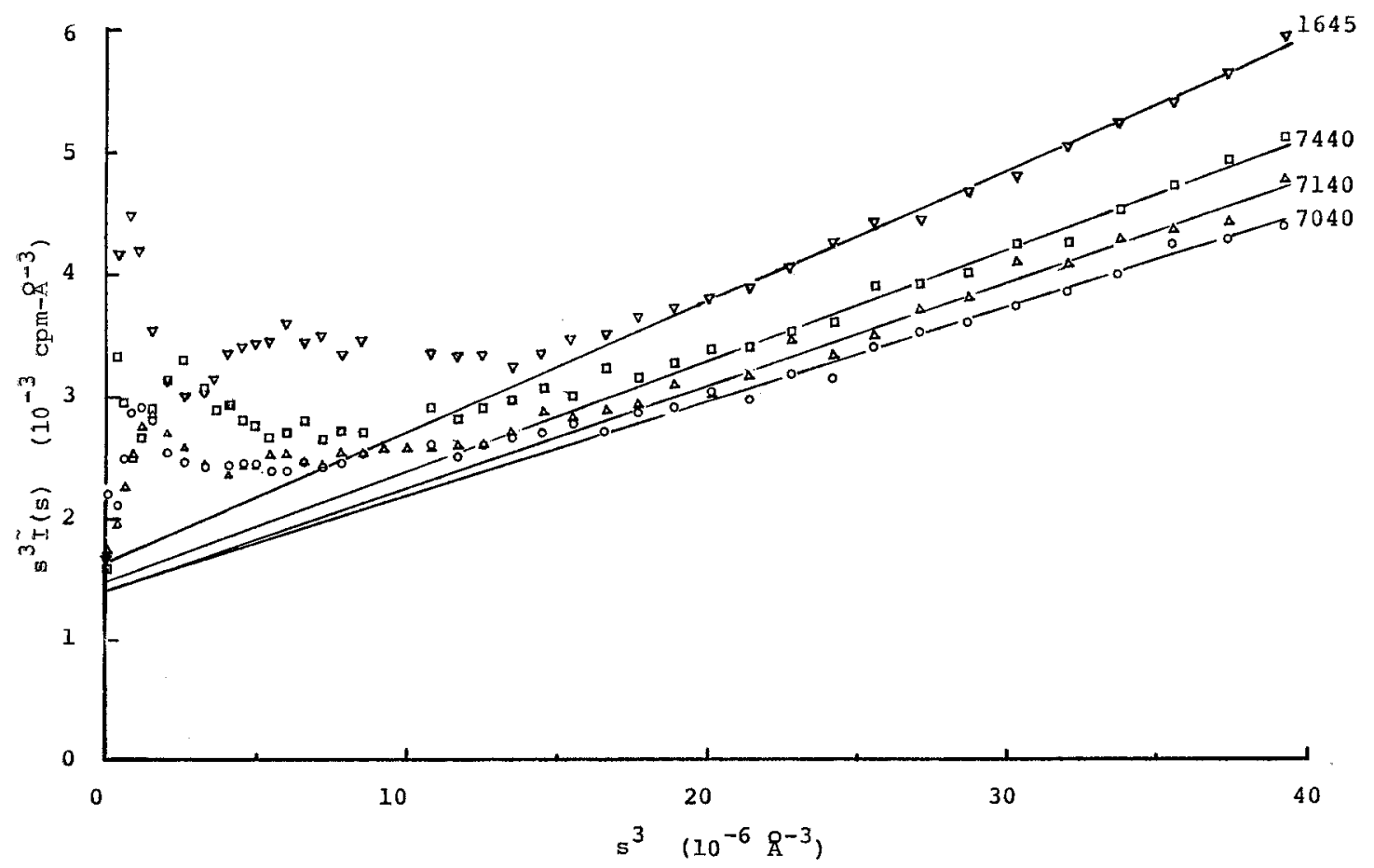

Fig. 8. Plots of $s^{3} I(s)$ vs $s^{3}$ for unirradiated PES 


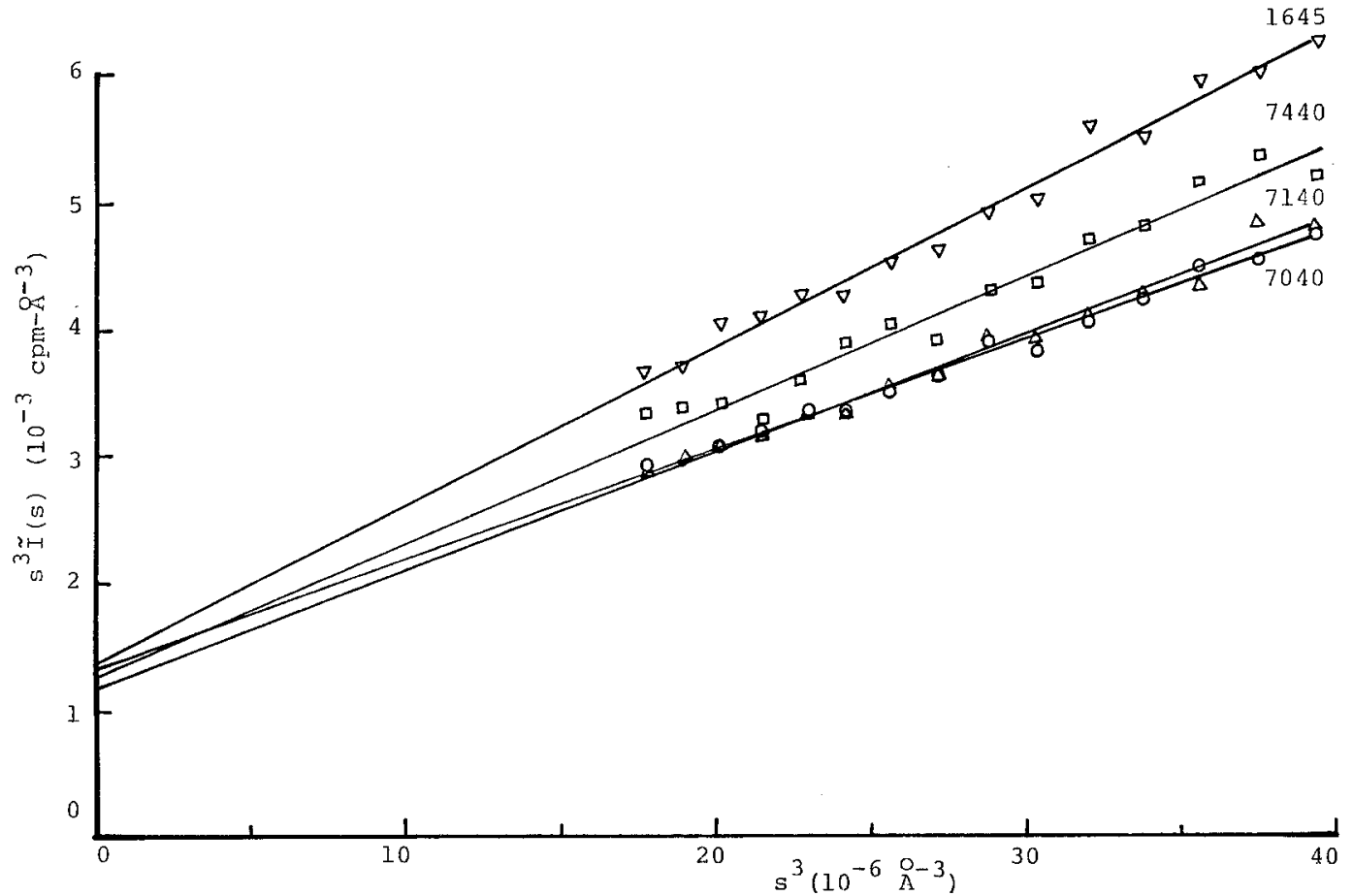

Fig. 9. Plots of $s^{3} I(s)$ vs $s^{3}$ for irradiated PEs (100 Mrad)

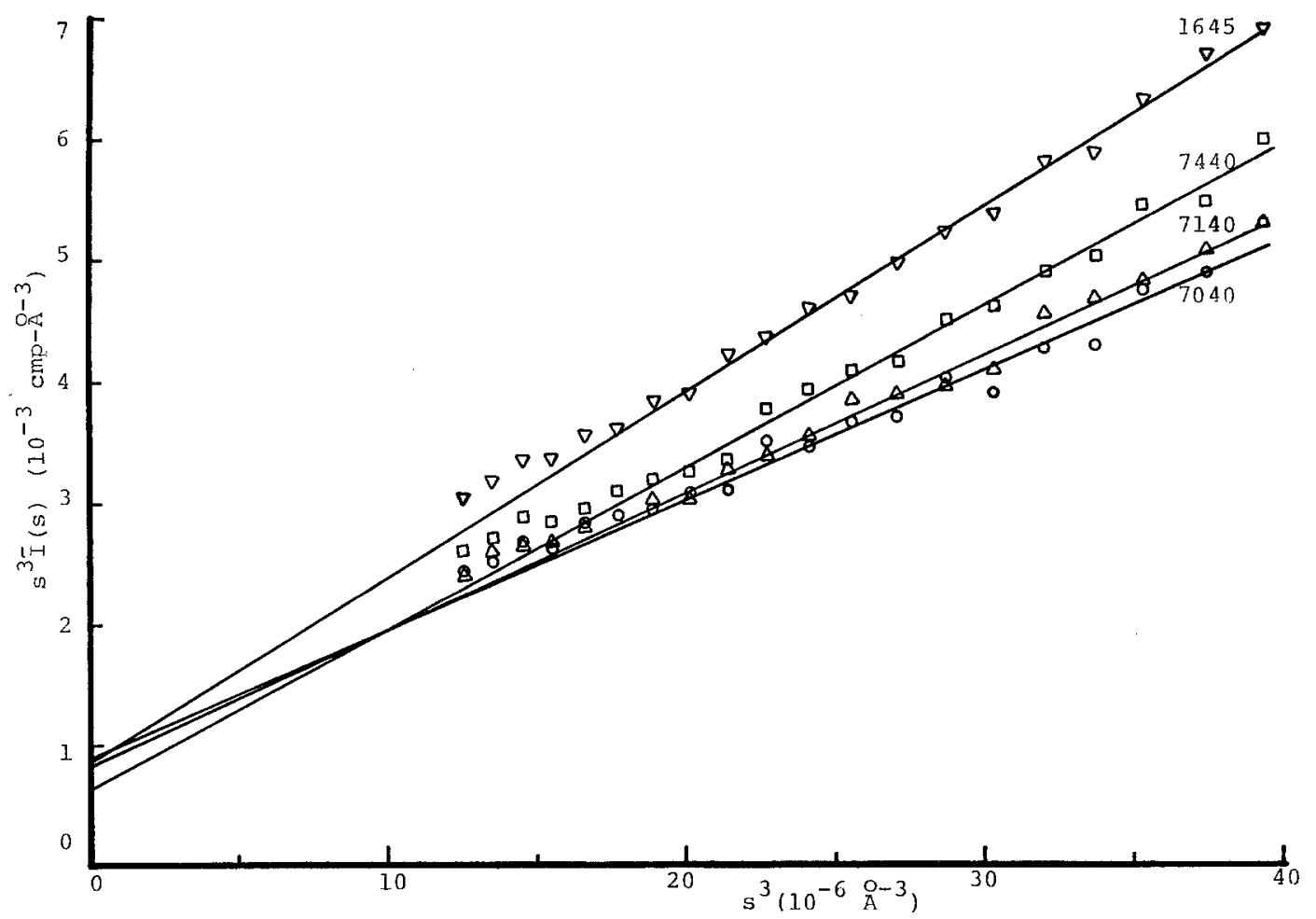

Fig. 10. Plots of $s^{3} I(s)$ vs $s^{3}$ for irradiated PEs (312 Mard) 


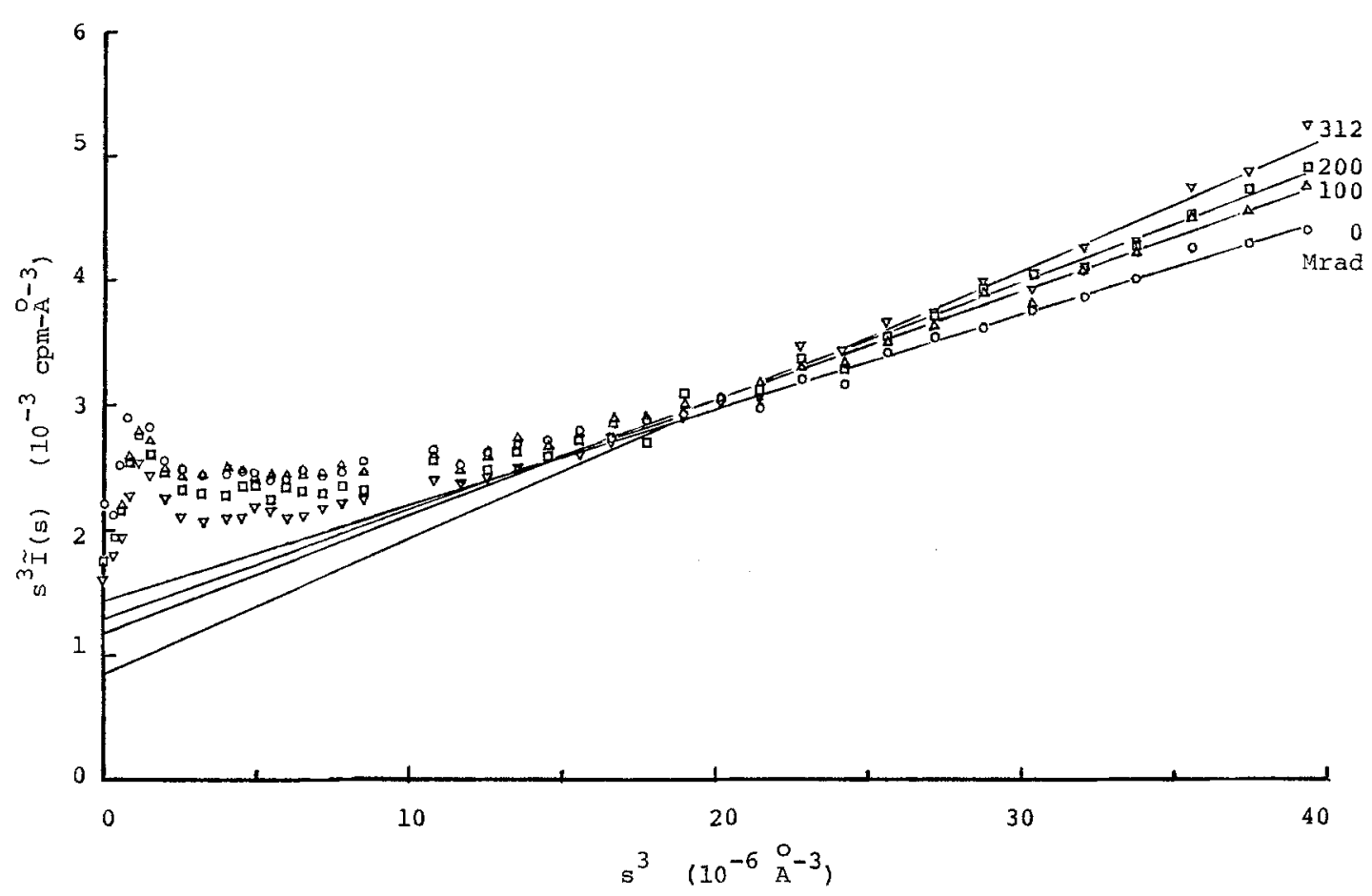

Fig. 11. Plots of $s^{3} I(s)$ vs $s^{3}$ for Alathon 7040 with various radiation doses

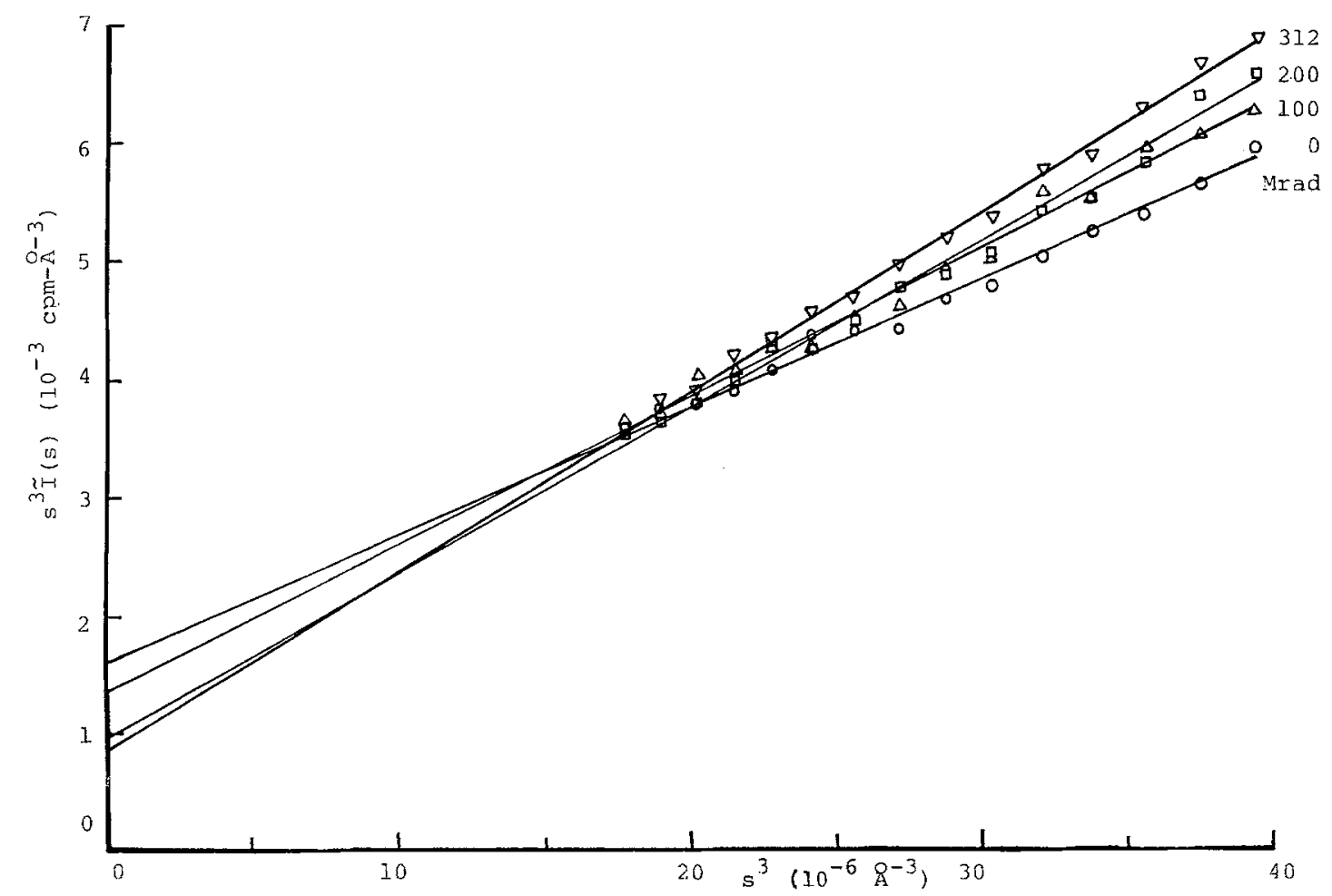

Fig. 12. Plots of $s^{3} I(s)$ vs $s^{3}$ for Alathon 1645 with various radiation doses 


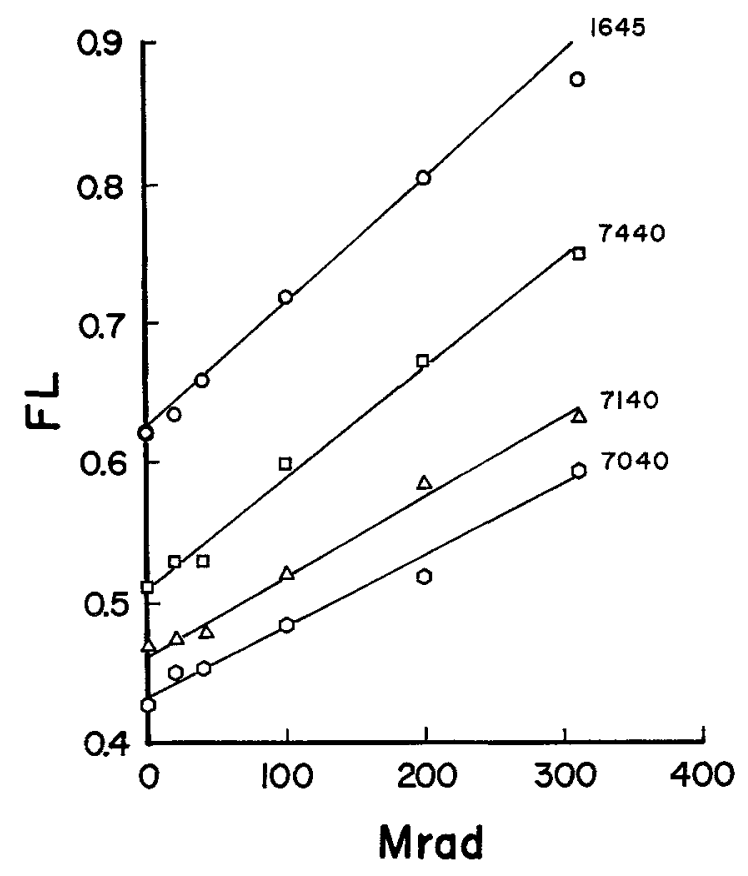

Fig. 13. Changes in density fluctuations as a function of radiation dose

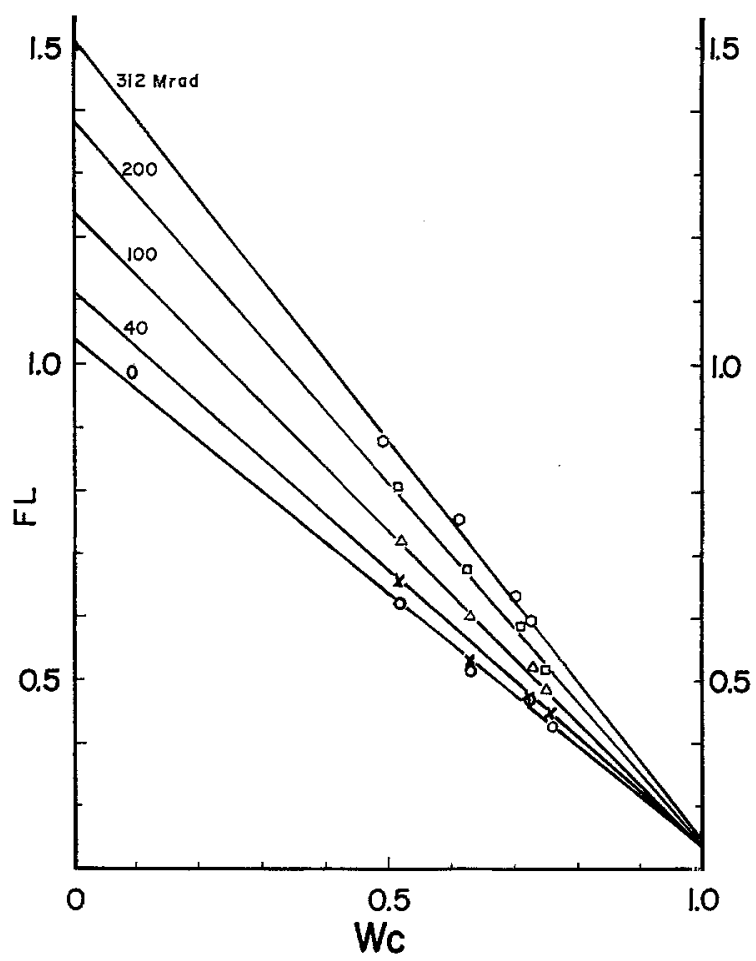

Fig. 14. Changes in density fluctuations as a function of crystallinity

\section{Discussion}

In general, the density fluctuation can be considered a disorder parameter; the more disorder there is, the higher the density fluctuation. For a perfect crystal lattice without thermal vibrations the density fluctuation is zero. Introduction of lattice defects can cause density fluctuation, which increases with increasing number and size of defects. The density fluctuation of an amorphous material, however, is determined by chain conformations and the state of local intermolecular order in the material.

In the case of semicrystalline polymers the density fluctuation consists of crystalline and amorphous components, $F L_{c}$ and $F L_{a}$. Since the chain molecules are less well-packed in the amorphous phase than in the crystalline phase, the density fluctuation in the amorphous phase is expected to be greater than in the more ordered crystalline phase. This is shown by a value of 0.23 for $F L_{c}$ and 1.04 for $F L_{a}$ in unirradiated PE samples. In addition, at a given dose, values of $F L_{a}$ are higher than $F L_{c}$, as shown in Figure 14. The value of 0.23 for $F L_{c}$ in unirradiated $P E$ represents the level of lattice defects inherent in crystals, which results in a lowering of $\rho_{c}\left(0.993 \mathrm{~g} / \mathrm{cm}^{3}\right.$ determined in the previous paper [2]) as compared to the ideal crystalline density $\left(0.998 \mathrm{~g} / \mathrm{cm}^{3}\right)$. The higher $F L_{a}$. value could be related to the degree of inhomogeneity in the noncrystalline phase [11]. Values of density fluctuation between 0.69 and 0.85 have been reported for unirradiated amorphous polymers [4,5].

Upon irradiation, additional density fluctuation is expected to be introduced by crosslinks, resulting in an increase in density fluctuations with radiation. This is clearly seen in all the samples. Furthermore, the increase in density fluctuation is found to be linear with radiation dose. At $312 \mathrm{Mrad}$ the increase in $F L$ from $0 \mathrm{Mrad}$ are 0.16 for Alathon 7040 and 0.26 for Alathon 1645.

The increase in density fluctuation with radiation can either be due to an increase of lattice defects introduced by crosslinks within the crystalline phase, or to the change in local density in the vicinity of the crosslinks in the amorphous phase. To determine which case is true, the fluctuations in each phase are resolved by extrapolation, as in the case of the unirradiated samples. Our results show that $F L_{a}$ is greater than $F L_{c}$ for each dose. Of particular significance is the finding that, for a given dose, the increase in $F L_{a}$ is much greater than that in $F L_{c}$ with respect to the corresponding initial values of $0 \mathrm{Mrad}$. The latter 
shows only a negligible increase of 0.01 at $312 \mathrm{Mrad}$. This indicates that no additional crystalline defects are introduced in the crystalline phase by crosslinks, in agreement with the conclusion reached in previous papers [1,2] which is based on the relative constant values of crystalline density $\rho_{c}$ as a function of degree of crosslinking. At the same dose (312 Mrad) an increase of 0.47 was obtained for $F L_{a}$. The increase in $F L_{a}$ with radiation can be attributed to the increasingly greater inhomogeneity due to the change in local density in the vicinity of crosslinks in the noncrystalline or amorphous phase, or most likely in the lateral grain boundary areas, as suggested previously $[1,2])$.

The use of $s^{3} \tilde{I}(s)$ vs $s^{3}$ plot in the determination of density fluctuation also provides the information of $\left\langle\eta^{2}\right\rangle$ which was determined from the "invariant" $Q$ in the previous paper [2]. The intercept of the plot is related to $\left\langle\eta^{2}\right\rangle$ as shown in Eq. (2). The values of intercept were found to decrease with increasing radiation dose (Figs. 11 and 12) for a given sample. This suggests that $\left\langle\eta^{2}\right\rangle$ decreases with increasing dose, in agreement with the results obtained in that paper.

According to Eq. (2), the value of $\left\langle\eta^{2}\right\rangle$ can be calculated from the intercept if the correlation length , $l_{c}$ is known. $l_{c}$ is related to the specific surface $S_{s p}$ by [12]

$$
S_{s p}=\frac{S}{V}=\frac{4 v_{c}\left(l-v_{c}\right)}{l_{c}},
$$

where $S / V$ is the surface area per unit volume and $v_{c}$ is the volume fraction of the crystalline phase. Assuming an even and parallel interface between the phases, $S_{s p}$ is related to the long period $L$ as [8]

$$
S_{s p}=\frac{2}{L}
$$

Therefore, $l_{c}$ can be calculated from Eqs. (6) and (7) using $L=275 \AA$ determined from the peak position of the SAXS curves of Alathon 7040. Values of $l_{c}$ are listed in Table $5 . l_{c}$ remains nearly unchanged, 109$114 \mathrm{~A}$, for all radiation doses. Table 5 also lists the values of intercept and $\left\langle\eta^{2}\right\rangle$ calculated from the intercept (shown as $\left\langle\eta^{2}>_{\text {int }}\right.$ ) for Alathon 7040 . For comparison, values of $\left\langle\eta^{2}\right\rangle$ determined from the "invariant" $Q$ in the previous paper [2] are also included in the table (shown as $\left\langle\eta^{2}>_{Q}\right.$ ). It shows that the values obtained from the two methods are in reasonable agreement, though $\left\langle\eta^{2}\right\rangle_{\text {int }}$ is a little greater than $\left\langle\eta^{2}>_{Q}\right.$ ). This could be due to the fact that, in practical cases, the interface is usually not perfectly even and parallel. These deviations will cause a larger specific surface, i.e., $S_{s p}>2 / L$. Therefore, $l_{c}$ will be smaller than the calculated values, which would lead to lower $\left\langle\eta^{2}\right\rangle_{\text {int }}$ values and, hence, better agreement between $\left\langle\eta^{2}>_{\text {int }}\right.$ and $\left\langle\eta^{2}\right\rangle_{Q}$ ).

Table 5. Alathon 7040

\begin{tabular}{llllllll}
\hline Mrad & 0 & 10 & 20 & 40 & 100 & 200 & 312 \\
\hline Intercept & & & & & & & \\
$\left(10^{-3} \mathrm{cpm} / \AA^{3}\right)$ & 1.441 & 1.330 & 1.390 & 1.396 & 1.318 & 1.200 & 0.887 \\
$l_{c}(\AA)$ & 109.1 & 109.5 & 110.2 & 108.9 & 111.0 & 111.5 & 113.9 \\
$<\eta^{2}>_{\text {int. }}^{*}$ & 1.538 & 1.424 & 1.498 & 1.487 & 1.431 & 1.309 & 0.988 \\
$\left\langle\eta^{2}>Q\right.$ & 1.517 & 1.463 & 1.439 & 1.351 & 1.320 & 1.244 & 1.136 \\
\hline
\end{tabular}

* Calculated from the intercept of $s^{3} \tilde{I}(s)$ vs $s^{3}$ plot; in (mole-electrons $\left./ \mathrm{cm}^{3}\right)^{2}$.

"Determined from the "invariant" $Q$ (in [2]); in (mole-electrons $\left./ \mathrm{cm}^{3}\right)^{2}$.

\section{Conclusions}

Density fluctuation was determined by using SAXS and PEs with varying radiation doses. It increases with decreasing crystallinity and with increasing radiation dose linearly. By means of extrapolation, the crystalline and amorphous density fluctuations, $F L_{c}$ and $F L_{a}$, were obtained. At a given dose, $F L_{c}$ is smaller than $F L_{\alpha}$. The increase in $F L_{a}$ with radiation is much greater than that of $F L_{c}$ compared with the initial values at 0 Mrad. Only a negligible increase in $F L_{c}$ was found at $312 \mathrm{Mrad}$. Based on these results it is concluded that no crosslinks are introduced within the crystalline phase. Therefore, crosslinks take place primarily in the noncrystalline phase, in agreement with the conclusions reached in the previous papers.

\section{Acknowledegment}

The authors wish to express their gratitude to the Macromolecular Research Center and the National Science Foundation for financial support.

\section{References}

1. Yeh GSY, Chen CJ, Boose DC (1985) Colloid \& Polymer Sci 263:109

2. Chen CJ, Boose DC, Yeh GSY (1990) ibid. etc., submitted for publication to Colloid \& Polymer Sci 
3. Guinier A, Fournet G (1955) Small-Angle Scattering of X-rays, Wiley, New York

4. Wendorff JH, Fischer EW (1973) Kolloid Z Polym 251:876

5. Ratbje I, Kuland W (1976) Colloid \& Polym Sci 254:358

6. Porod G (1951) Kolloid Z. A., 124:83

7. Ruland W (1971) J Appl Cryst 4:70

8. Perret R, Ruland W (1971) Kolloid Z. Polym 247:835

9. Levelut AM, Guinier A (1967) Bull Soc Miner Crist 40:445

10. Hendrick RW, Schmidt PW (1967) Acta Phys Austriaca 26:97; 37:20 (1973)

11. Harget PJ, Siegmann A (1972) J Appl Phys 43:4357

12. Debye $P$, Anderson HR, Bueche A (1957) J Appl Phys 28:679

Authors' address:

Prof. G. S. Y. Yeh

Dept. of Chemical and Metallurgical Engineering

Macromolecular Research Center

University of Michigan

Ann Arbor, Michigan 48107, USA

Received May 14, 1990

accepted June 20, 1990 